\title{
Performance Evaluation of Wireless Sensor network for High Data Rates
}

\author{
[ TehminaKaramat Khan, Taimur Karamat, Zulkefli Bin Muhammed Yusof ]
}

\begin{abstract}
WSN multimedia services are experiencing performance related issues like packet loss, delay etc due to constrained resources. The need to develop solutions that optimize the utilization of resources, improve performance, reduce packet loss and enhance the QoS requires performance evaluation of WSN where packets arrive and depart the service facility (sink) in burst. In this paper we have developed a novel analytical solution where packets from sensor nodes arrive in burst and after getting service depart the service facility in burst. The evaluation is carried out by varying standard coefficient of variance for inter service time and inter arrival time and by varying utilization of the sink node. The numerical results proves the credibility of the solution devised and useful results are deduced.
\end{abstract}

Keywords- Wireless sensor networks; Quality of service; Queuing Network Models; advance future wireless sensor networks.

\section{Introduction}

This Rapid Technological progress in micro-electromechanical systems (MEMS) and radio components enabled a new generation real-time embedded Wireless Sensor Networks (WSNs) [1, 2]. These devices communicate with each other over physical environment, perhaps for a short duration, with common capabilities of distributed sensing, storing and for collaboration in a more convenient manner. These devices can be disseminated under extreme environment and can act as monitoring shield at a safer distance. The research is motivated by the fact that utilization efficiency of wireless sensor networks (WSN) is enormous in many fields such as surveillance, industrial and consumer applications, health monitoring, and so on $[3,4,5]$ whereas on the other hand the lack of proper thoroughly evaluated communication protocols is a major factor in its slow and restricted growth. Due to remote deployment of WSNs, the major challenge is the power supply [6,7] since lifetime of the sensor nodes will shorten, if there is any imbalance in energy consumption across the network due to premature failure of devices [8-12]. The primary focus of WSNs network is different from traditional wireless networks such as Adhoc and cellular wireless networks. The necessary goal of interest is to optimize constraints on quality of services such as computational speed, bandwidth, signal-noise distortion ratio, delays and loss.

Transmission of data through multimedia WSNs demands high communication bandwidth and reliability for better collection of largely chunked data. Though, some applications of WSNs beside aforementioned characteristics require high quality. In this scenario, major factor in quality of service (QoS) metrics in WSNs is in reliability of network and quality. For interfacing between sensors and energy, selecting relevant microcontroller component and transceiver plays an important role, where data controls should be organized in such a way that incoming data packet loss is minimized. The scheme of multimedia traffic in WSN with Quality of Service (QoS) assures the required performance and analysis modeling of nodes of Queuing Network Models (QNMs). This paper emphasizes on performance evaluation of a non-preemptive scheme for high data rates in WSN, given Blocking probability (PB) will illustrates clearly that how system specification be selected so that blocking state could be minimized.

To evaluate the WSN the packets arrive in burst and depart in burst, we have first derived analytical expressions for performance evaluation, followed by numerical results

\section{Related Work}

To minimize packet loss in [1] buffering mechanism was improved in MAC layer so that the arriving packets may be prevented by minimizing retransmission of packets. It was proposed that relevant transceivers and microcontroller equipment be designed to minimize packet loss data controls with out compromising QoS. In [2] an adaptive queue management scheme is proposed that constraints the average queuing delay in a router to a required value through the mapping relationship between queuing delays and queuing thresholds. Packets are dropped when the queue length reaches the threshold. In [26] a priority based scheme is proposed where the packets with higher priority are transferred at higher data rates and low priority packets are transferred with low data rates to minimize end to end delay. The model is for single arrival and single departure of packets and follows poisson distribution. In [25] the authors carried out performance evaluation for buffer less queue for WSN, an analytical solution was devised to analyze the blocking of packets. The packet arrival process is poison and follow single arrival single departure phenomena. The results shows that the blocking of packets increase with the increase in arrival rate.

We have proposed an analytical solution followed by numerical results where traffic arrival and departure is in burst. The packets arrive from different nodes to sink node in batches and leave the sink node after getting processed in batches. The blocking of packets is calculated by varying Squared co efficient of variance for inter arrival and inter service times and by increasing the utilization of sink node

\section{Model Assumptions}

To derive the analytical solution the behavior of traffic is modeled. We assume the case that no packets leave without service when the system is empty and packets arrive at the sink node get service. Also when packet arrive at the sink node and the queue is full; the packets will be blocked and these lost packets leave the system and never return to the system again. Due to high band width and high data rates the packets do arrive at the sink node in burst and are serviced in burst. These packets follow generalized exponential distribution for arrival process and batch poisson distribution for service. The analytical expressions for blocking, number 
Proc. of the Fourth Intl. Conf. Advances in Computing, Communication and Information Technology- CCIT 2016

Copyright (C) Institute of Research Engineers and Doctors, USA .All rights reserved.

ISBN: 978-1-63248-092-7 doi: 10.15224/ 978-1-63248-092-7-66

of packets processed, number of packets arrived and number of packets in service are derived by taking the limit of first branch of receiver and server to infinity of Two stage hyper exponential model. The description of the model is as follow

The inter arrival and inter service time for two state hyper exponential model are independent and exponentially distributed with mean $1 / \lambda \_2$ at receiver centre branch and $1 / \mu 2$ at service centre branch respectively. A packet that enters the system can either choose branch 2 with probability $1-\sigma$ or with probability $\sigma$ can choose second branch, and at the server centre can choose branch 2 or 1 with probability $1-\gamma$ or with probability $\gamma$ respectively as shown in Figure $1(\mathrm{a})$.

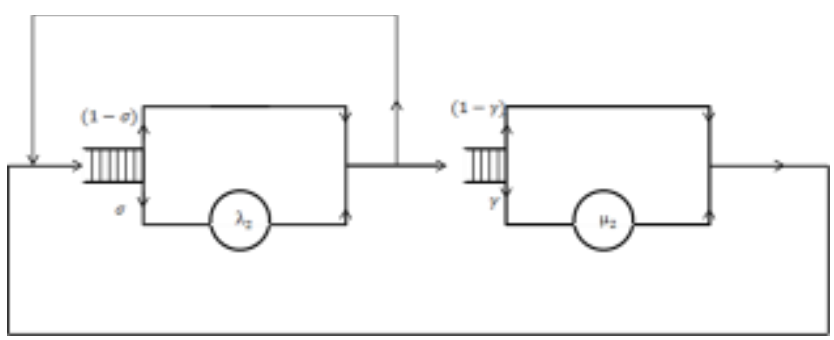

Figure 1(a) 1(b)

State transition diagram of system is shown in Figure

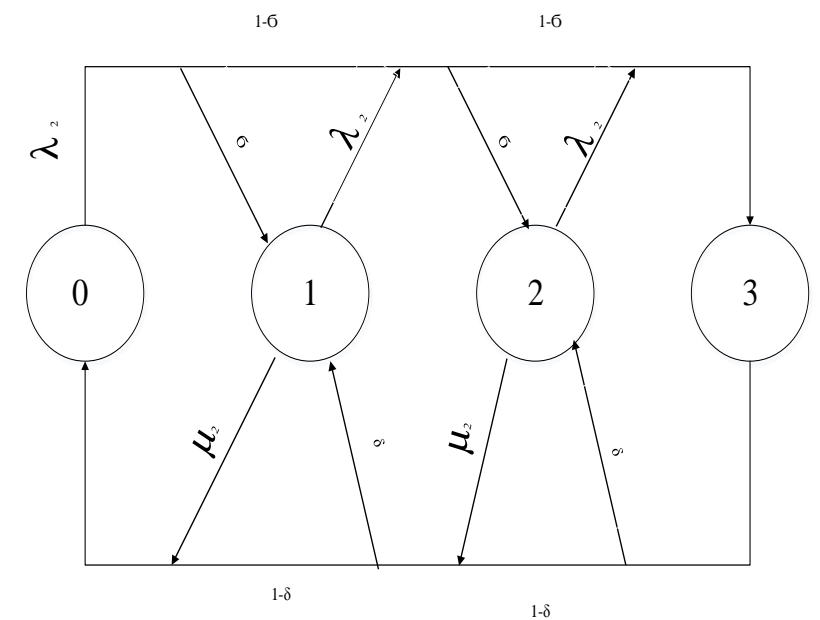

Figure 1(b):

\section{Iv. Flow Balance Equations}

$$
\begin{aligned}
& \lambda_{2} \mathrm{p}(0)=\mu_{2} \mathrm{p}(1)+(1-\gamma) \mu_{2} \mathrm{p}(2)+(1-\gamma)^{2} \mu_{2} \mathrm{p}(3) \ldots \text { (i) } \\
& \left(\lambda_{2}+\mu_{2}\right) \mathrm{p}(1)=\lambda_{2} \sigma \mathrm{p}(0)+\gamma \mu_{2} \mathrm{p}(2)+\gamma(1-\gamma) \mu_{2} \mathrm{p}(3) \text { (ii) } \\
& \left(\lambda_{2}+\mu_{2}\right) \mathrm{p}(2)=\lambda_{2}(1-\sigma) \sigma \mathrm{p}(0)+\lambda_{2} \sigma \mathrm{p}(1)+ \\
& \gamma \mu_{2} \mathrm{p}(3) \ldots \text { (iii) }
\end{aligned}
$$

$\mu_{2} \mathrm{p}(3)=\lambda_{2}(1-\sigma)^{2} \mathrm{p}(0)+\lambda_{2}(1-\sigma) \mathrm{p}(1)+$ $\lambda_{2} \mathrm{p}(2) \ldots$...(iv)

By solving aggregate global balance equations (i), (ii), (iii) and (iv), for tangible states, single step Birth \& Death model is deduced. State probabilities are as follows

$\mathrm{p}(1)=\frac{\lambda_{2}(\sigma+\gamma(1-\sigma))}{\left(\lambda_{2}(1-\gamma)+\mu_{2}\right)} \mathrm{p}(0)$

$p(2)=\frac{\left(\lambda_{2}+(1-\sigma) \mu_{2}\right)}{\left(\lambda_{2}(1-\gamma)+\mu_{2}\right)} p(1)$

$\mathrm{p}(3)=\frac{\left(\lambda_{2}+(1-\sigma) \mu_{2}\right)}{\mu_{2}(\sigma+\gamma(1-\sigma))} \mathrm{p}(2)$

GE Inter arrival and service time are independent and identically distributed according to GE distribution and geometrically distributed batches are of renewal one [5], therefore mean bulk arrival rate is $\sigma \lambda$ and mean bulk departure rate is $\gamma \mu$. By substituting $\lambda \_2$ with $\sigma \lambda$ and $\mu \_2$ with $\gamma \mu$, we get solution for censored GE/BPP/1/N as follows

$$
\begin{aligned}
& \mathrm{p}(1)=\frac{\rho \sigma(\sigma+\gamma(1-\sigma))}{\rho \sigma(1-\gamma)+\gamma} \mathrm{p}(0) \quad \ldots \ldots \ldots \ldots . . \mathrm{i} \\
& \mathrm{p}(2)=\frac{\rho \sigma+(1-\sigma) \gamma}{\rho \sigma(1-\gamma)+\gamma} \mathrm{p}(1) \quad \ldots \ldots \ldots \ldots . \mathrm{ii} \\
& \mathrm{p}(3)=\frac{\rho \sigma+(1-\sigma) \gamma}{\gamma(\sigma+\gamma(1-\sigma))} \mathrm{p}(2) \quad \ldots \ldots \ldots \ldots . . \mathrm{iii}
\end{aligned}
$$

State transition diagram is given in figure

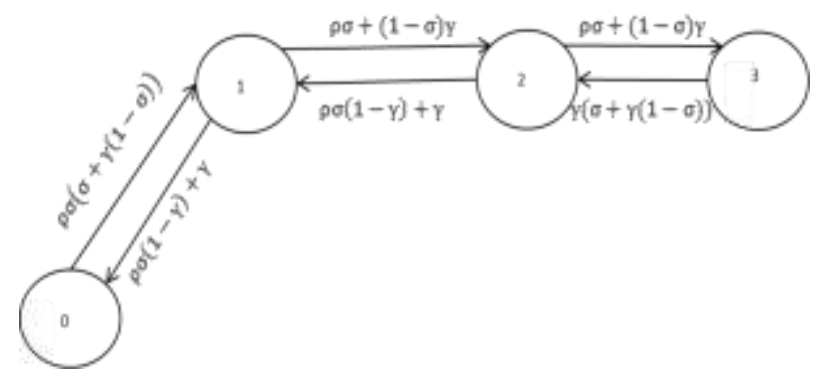

Figure 2(a): GE/BPP/1/3 Global balance Diagram

System can be now extended to $\mathrm{N}$ number of customer. State transition diagram for $\mathrm{N}$ customers is shown in figure

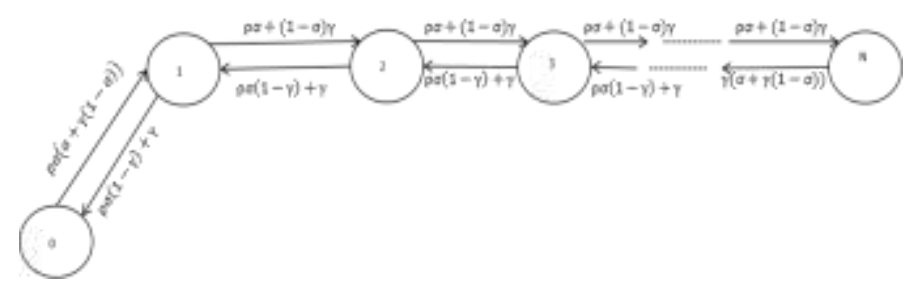

Figure 2(b): GE/BPP/1/N Global balance Diagram 
Proc. of the Fourth Intl. Conf. Advances in Computing, Communication and Information Technology- CCIT 2016

Copyright (C) Institute of Research Engineers and Doctors, USA .All rights reserved.

ISBN: 978-1-63248-092-7 doi: 10.15224/ 978-1-63248-092-7-66

The performance measures in terms of squared coefficient of variation we substitute $\delta=\left(\frac{2}{1+C_{a}^{2}}\right) \& \gamma=\left(\frac{2}{1+C_{s}^{2}}\right)$ in equation i, ii and iii.

$a=\frac{2 \rho\left(c s^{2}+c a^{2}\right)}{\left(c a^{2}+1\right)\left(\rho c s^{2}+c a^{2}-\rho+1\right)}$

$\mathrm{b}=\frac{\rho c s^{2}+c a^{2}+\rho-1}{\rho c s^{2}+c a^{2}-\rho+1}$

$\mathrm{c}=\frac{\left(c s^{2}+1\right)\left(\rho c s^{2}+c a^{2}+\rho-1\right)}{c s^{2}+c a^{2}}$

$p(1)=a \cdot p(0)$

$$
p(2)=a \cdot b \cdot p(0)
$$

$p(3)=a \cdot b^{2} \cdot p(0)$

$p(N-1)=a \cdot b^{n-2} \cdot p(0)$

$p(N)=a \cdot b^{n-2} \cdot c \cdot p(0)$

$p(0)=\frac{1}{\left(a+a \cdot b+a b^{2}+\ldots \ldots \ldots+a b^{N-2}+a b^{N-2} c\right.}$

OR

$\mathrm{p}(0)=\left(a+a \cdot b+a b^{2}+\ldots \ldots \ldots . .+a b^{N-2}+a b^{N-2} c\right)^{-1}$

Blocking $=1-P_{N}$

\section{v. Results}

To calculate numerical results for blocking by varying server utilization and SQV, following results has been obtained using Matlab.

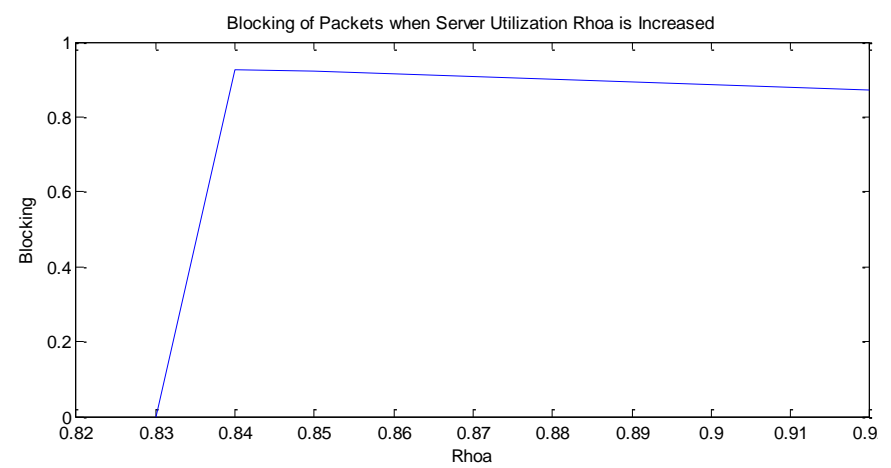

Figure 3(a)

Busy period also known as utilization of sink node i.e. time sink node is busy processing packets; also plays an integral part in calculating other statistics. The Figure 3(a) shows the impact of utilization on blocking of packets. We can see from the figure that as utilization of the sink node is increased the blocking of packets is decreased i.e. more packets are processed and the blocking of packets is decreased. Hence we can say that increasing the utilization will increase the performance and will decrease loss of packets. This will also optimize utilization of resources.

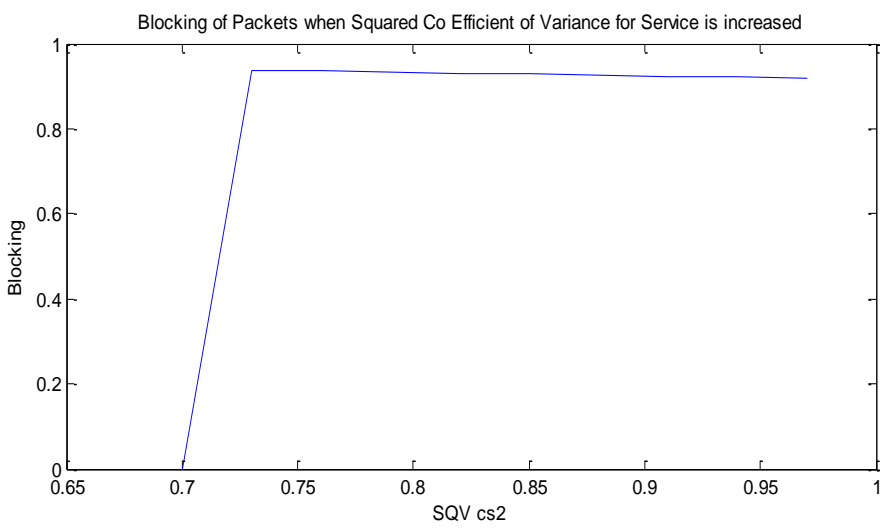

Figure 3(b)

The SQV is the squared coefficient of variance used to approximate general inter arrival time and service time with known first two moments. In the above Figure 3(b), SQV for arrival of packets is increased and we can see in the figure that the blocking of packets is decreased. At 0.7 the blocking of packets is between $0.8-1$ as the SQV for service time increases with interval 0.3 the blocking of packets eventually decreases and at 0.95 its nearly equal to 0.8 . It will be interesting to note when both SQV and utilization are put to increase and see how it effects blocking.

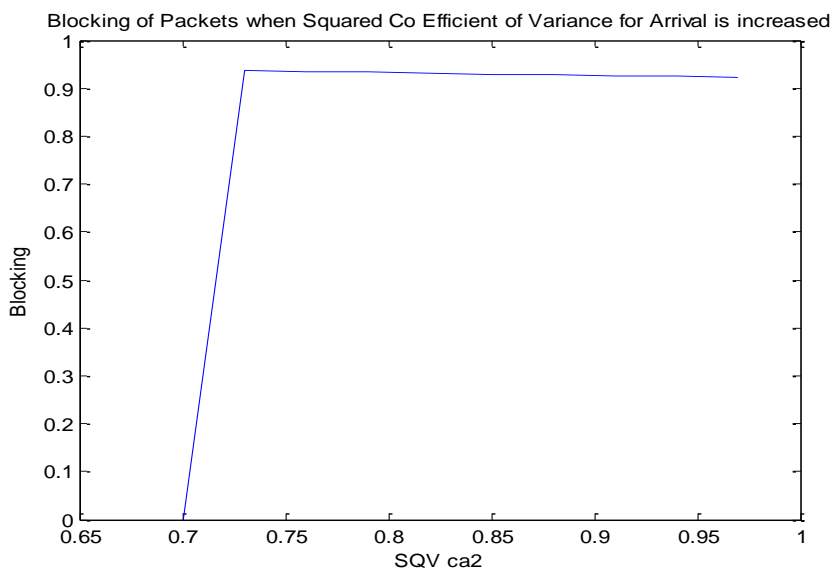

Figure 3(c)

The burstiness of the arrival process is characterized by Squared Coefficient of variance of the inter arrival time or equally the size of the incoming bulk of packets. From Figure 3(c) above we observed that with the increase in squared coefficient of variance of inter arrival time, blocking of packets is decreased.

\section{vi. Conclusion and Future work}

In this paper analytical solution was devised for WSN where packets arrive in burst, get service and depart the service facility in burst. The above results shows that with the increase in utilization the blocking of packets is reduced and with the increase in SQV for inter arrival and inter service time the blocking of packets is reduced. The results also indicate the credibility of analytical solution deduced in this paper. 
Proc. of the Fourth Intl. Conf. Advances in Computing, Communication and Information Technology- CCIT 2016

Copyright $\odot$ Institute of Research Engineers and Doctors, USA .All rights reserved.

ISBN: 978-1-63248-092-7 doi: 10.15224/ 978-1-63248-092-7-66

In this paper we have deduced analytical solution for bursty traffic for WSN to study the impact of increased utilization, SQV for inter arrival and service time of packets on blocking. In future we will work on multi types of packets i.e. voice, video and data and will assign priority to packets to investigate the performance of WSN network under bursty traffic.

\section{References}

[1] J. G. K. Sohrabi, V. Ailawadhi and G. Pottie, "Protocols for selforganization of a wireless sensor network. ," IEEE Personal Communications, pp. 16-27, October 2000.

[2] M. J. A. J.M. Rabaey, J.L.D Silva,D.Patel,S.Roundy., "Pico Radio supports ad hoc ultra low power wireless networking," IEEE Computer. pp., pp. 42-48, July 2000.

[3] K. L. J.W.Allen, M.Welsh,O.Marcillo,J.Johnson,M.Ruiz,J.Lees. "Deploying a wireless sensor Network on an active volcano.," IEEE Internet Computing, pp. 18 - 25, April 2006.

[4] K. C. J.Peak, R.Govindan, J.Caffrey, S.Marsi "A Wireless Sensor Network for Structural Health Monitoring: Performance and experience," In Proc. 2nd IEEE Workshop on Embedded Networked Sensors (EmNetS-II). pp. 1-10, May 2005.

[5] P. J. N. G.A. Cranch, C.K. Kirkendall., "Large-scale remotely interrogated arrays of fiber-optic interferometric sensors for underwater acoustic applications," IEEE Sensors Journal, pp. 19-30, Feburary 2003.

[6] W. S. I.F. Akyildiz, Y. Sankarasubramaniam, E. Cayirci., "A Survey on Sensor Networks.," IEEE Communications Magazine, pp. 102-114, August 2002.

[7] C. C. a. S. P. Kumar., "Sensor Networks: Evolution, Opportunities, and Challenges.," In Proeedings of. IEEE, pp. 1247-1256, August 2003.

[8] Q. Z. a. L.Tong., "Energy efficiency of large-scale wireless networks: proactive versus reactive networking.," IEEE Journal on Selected Areas in Communications., pp. 1100 - 1112, May 2005

[9] E. S. J.Arias, I.Marin,J. Jimenez,J. Lazaro and A. Zuloaga., "Node Synchronization In Wireless Sensor Networks.," In IEEE International Conference on Wireless and Mobile Communications (ICWMC). pp. $50-55$, July 2006.

[10] R. C. S. a. J. M. Rabaey., "Energy Aware Routing for Low Energy Ad Hoc Sensor Networks.," In IEEE Wireless Communications and Networking Conference (WCNC). pp. 350- 355, March 2002

[11] I. K. a. M. Kandemir., "Tuning In-Sensor Data Filtering to Reduce Energy Consumption in Wireless Sensor Networks.," In Proceedings of the conference on Design, automation and test Europe by IEEE Computer Society,, pp. 852 -857, February 2004.

[12] C. K. a. S. K. S.Lee, "Constructing Energy Efficient Wireless Sensor Networks by Variable Transmission Energy Level Control.," In Proceedings of the Sixth IEEE International Conference on Computer and Information Technology (CIT'06), pp. 225-231, September 2006.

[13] S. F. a. P. S. O.Younis, "Robust communications for sensor networks in hostile environments.," In 12th IEEE Internatioanl Workshop on Quality of Service(IWQOS). pp. 10 - 19, June 2004.

[14] M. Y. C.Li, G.Chen and J.Wu. , "An energy-efficient unequal clustering mechanism for wireless sensor Networks.," In IEEE International Conference on Mobile Adhoc and Sensor Systems Conference., pp. 7-10, November. 2005.

[15] A. E. K. J.N.Karaki, "Routing techniques in wireless sensor networks: a survey.," IEEE Wireless Communications., pp. 6- 28, December 2004

[16] S. F. 0. Younis, "HEED: A Hybrid, Energy-Efficient, Distributed Clustering Approach for Ad Hoc Sensor Networks.," IEEE Transactions on Mobile Computing, pp. 366-379., December 2004.

[17] N. I. a. I.U.Awan., "Coverage Based Inter cluster Communication for Load Balancing in Wireless Sensor Networks.," In the Proceedings of the 21st IEEE International Conference on Advanced Information Networking and Applications Workshops, pp. 923-928, June 2007.
[18] N. I. a. I. U. Awan, "Lower Bound for a Hierarchal Grid based wireless sensor networks," In the 23rd UK Performance Engineering Workshop, pp. 20-25, July 2007.

[19] C.Bettstetter., "On the minimum node degree and connectivity of a wireless multi hop network," In the Proceedings of the 3rd ACM international symposium on Mobile ad hoc networking and computing, , pp. 80 - 91, June 2002.

[20] C.Bettstetter., "On the connectivity of wireless multi hop networks with homogeneous and inhomogeneous range Assignment," In the IEEE Vehicular Technology Conference, pp. 1706 - 1710. , September 2002

[21] X. M. L.Yu; N.Wang, "Real-time forest fire detection with wireless sensor networks.," In the IEEE International Conference on Wireless Communications, Networking and Mobile Computing. , pp. 1214 -1217 , September 2005

[22] I. U. A. N.Israr, "Coverage Based Inter cluster Communication for Load Balancing in heterogeneous Wireless Sensor Networks," Journal of Telecommunication Systems (2008) 38: 121-132: Springer 2008.

[23] W. K. G. Seath, H.Y.Lee, P.Sun., "Energy Implication of clustering in heterogeneous Wireless Sensor Networks - An Analytical view.," In the 17th Annual IEEE International Symposium on Personal, Indoor and Mobile Radio Communication., pp. 1-5, September 2006.

[24] [25] Agassi Melikov,Anar Rustamov,"Queuing Management in wireless sensor networks for QOS measurement" Published online wireless sensor networks,2012,4,211-218.

[25] [26] Mary Cherian,T.R Gopal A Krishnan Nair"Priority Based Bandwidth Allocation in wireless sensor networks" International Journal of computer networks and communication,Vol 6,No6 Nov 2014.

[26] [27] Fuad Bajaber, Irfan Awan “ Adaptive dececntralized re-clustring protocol for wireless sensor networks" published in journal of computer and system sciences 77(2011)282-292.

[27] [28] Shahin Mahdizadeh Aghdam,Mohammad Khansari,Hamid R

[28] Rabiee, Mostafa Salehi "WCCP: A congestion control protocol for wireless multimedia communication in sensor networks" published in AdHoc Networks 13(2014)516-534.

[29] [29]L.B Lim,L.Guan,A.Grigg,I.W.Phillips,X.G.Wang,I.U.Awan "Controlling mean queuing delay under multi-class bursty and corelated traffic"published in journal of computer and system sciences 77(2011)898-916.

\section{About author(s):}

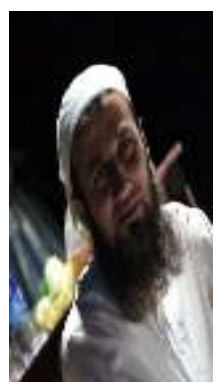

Tehmina Karamat $\mathrm{PhD}$ scholar(KICT) at international Islamic University Malaysia and is working as Assistant Professor in software engineering department at foundation University Islamabad Pakistan. Research areas are routing and queue management of wireless networks based on queuing theoretic concepts and information theoretic principles of maximum entropy.

TaimurKaramat is working as Instructor in Virtual University of Pakistan, administering Advanced Computer Networks course and had devised new and cost effective solutions for 2-D markovian and bursty multi traffic queues based on queueing theoretic concepts and information theoretic principles of maximum entropy

Zulkefli Bin Muhammed Yusuf. Is working as an Assistent Professor at KICT IIUM Gombak Campus Malaysia. His area of research are computer communication and information systems and high speed networks. 